\title{
Automedicación de medicamentos genéricos en usuarios de farmacias en un municipio mexicano
}

\section{Self-medication of generic drugs in users of pharmacies in a Mexican municipality}

\author{
María Jesús Almeida Cerino ${ }^{1}$, Heberto Romeo Priego Álvarez ${ }^{2}$, Juan Antonio Córdova Hernández ${ }^{3}$, \\ Manuel Higinio Morales García ${ }^{4}$, Pablo Sevilla Jerónimo 5
}

\section{Historial del artículo \\ Fecha de recepción: 16/01/2020}

Fecha de aprobación: 10/04/2020

1 Universidad Juárez Autónoma de Tabasco, Maestra en Salud Pública. Tabasco, México. ORCID https://orcid.org/0000-0002-1206-1885

2 Universidad Juárez Autónoma de Tabasco, Doctor en Medicina y Doctor en Ciencias de la Salud. Profesor Investigador, Tabasco, México. ORCID https://orcid.org/0000-0001-9217-5702

3 Universidad Juárez Autónoma de Tabasco, Doctor en Ciencias de la educación. Profesor en investigador, Tabasco, México. ORCID https://orcid.org/0000-0001-6138-0957

4 Universidad Juárez Autónoma de Tabasco, Doctor en Ciencias de la educación. Profesor en investigador, Tabasco, México. ORCID https://orcid.org/0000-0003-2560-6300

5 Universidad Juárez Autónoma de Tabasco, Estudiante de la Licenciatura de Médico Cirujano, Tabasco, México. ORCID https://orcid. org/0000-0003-0359-5540

Correspondencia: Heberto R. Priego Álvarez. Dirección: Centro de Investigación. División Académica de Ciencias de la Salud. Juárez Autónoma de Tabasco. Avenida Gregorio Méndez Magańa 2838/A Colonia Tamulté, C.P. 86150. Villahermosa, Tabasco, México. Correo electrónico: heberto_priego@hotmail.com

Como citar este artículo: Almeida-Cerino M, Priego-Álvarez HR, Córdova-Hernández J, Morales-García M, Sevilla-Jeronimo P. Automedicación de medicamentos genéricos en usuarios de farmacias en un municipio mexicano. Revista de la Facultad de Ciencias de la Salud de la Universidad del Cauca. 2020; 22 (1): 24- 32 


\section{RESUMEN}

Introducción: La automedicación es la utilización de medicamentos sin receta por elección propia de las personas, lo que puede condicionar riesgos a la salud y generar potencial resistencia antimicrobiana.

Objetivo: Identificar el nivel de conocimiento básico y las características de la automedicación en consumidores de medicamentos genéricos en farmacias de un municipio mexicano.

Métodos: Se realizó un estudio exploratorio descriptivo $y$ transversal en la población urbana residente en el Municipio de Jalpa de Méndez (87,249 habitantes) Tabasco. La muestra probabilística aleatoria simple ( $n=383)$ recolectó información de usuarios compradores de medicamentos genéricos en 7 de las 10 farmacias existentes en la cabecera municipal.

Resultados: Elperfil de los consumidores de medicamentos genéricos corresponde mayormente a mujeres (53\%), entre 18 y 33 ańos de edad (53.3\%), con nivel de escolaridad alta (preparatoria $34 \%$ y profesionales $42 \%$ ), casadas (45\%), ocupadas laboralmente (52\%) y autodependientes (62\%). El nivel de conocimiento predominante fue el medio. Los medicamentos mayormente comprados fueron antiinflamatorios no esteroideos comúnmente denominados AINES (46.3\%). La automedicación fue abiertamente reconocida por el $56.60 \%$ de los encuestados.

Conclusiones: La alta escolaridad se relaciona a la práctica de automedicación en la población estudiada. El patrón de automedicación detectado se caracteriza por la compra sin receta médica en el caso de patologías leves, principalmente debido a la falta de tiempo para acudir al médico y por los bajos costos de las especialidades farmacéuticas genéricas.

Palabras clave: Automedicación, Hábitos de consumo de medicamentos, Medicamentos genéricos, México.

\section{INTRODUCCIÓN}

Los medicamentos genéricos o especialidades farmacéuticas genéricas (EFG) son productos que han perdido su patente, presentan la misma composición cualitativa en principios activos con la misma forma farmacéutica (1) y pueden ser producidos y comercializados por diversos laboratorios. En

\section{ABSTRACT}

Introduction: Self-medication is the use of over-the-counter medications atpeople's choice, which can condition health risks and generate potential antimicrobial resistance.

Objective: To identify the characteristics of self-medication of generic drug consumers in pharmacies of a Mexican municipality

Methods: A descriptive and cross-sectional exploratory study was carried out in the urban population residing in the Municipality ofJalpa de Méndez (87,249 inhabitants), Tabasco. The simple random probabilistic sample $(n=383)$ collected information from users / buyers of generic drugs at 7 of the 10 pharmacies in the municipal capital.

Results: Theprofile of consumers of generic drugscorresponds mostly to women (53\%), between 18 and 33 years of age (53.3\%), with a high level of schooling (high school $34 \%$ and professionals $42 \%$ ), married (45\%), employed (52\%) and selfdependent (62\%). The predominant level of knowledge was the medium. Most commonly purchased medications were non-steroidal anti-inflammatory drugs commonly called NSAIDs (46.3\%). Self-medication was openly recognized by $56.60 \%$ of respondents.

Conclusion: Educational level is related with the practice of self-medication in the population studied. The selfmedication pattern detected is characterized by the purchase without a prescription in the case of mild pathologies, mainly due to the lack of time to go to the doctor, and the low costs of specialty generic drugs.

Key words: Self-medication, Drug use habits, Generic drugs, Mexico.
México se conocen como genéricos intercambiables y en otros países como productos similares (2).

La Federación Farmacéutica Internacional y la Industria Mundial de la Automedicación Responsable aceptan y presentan la definición de automedicación como la utilización de medicamentos sin receta, por elección propia de las personas $(3,4)$. Esto puede condicionar la resistencia 
antimicrobiana, que en palabras del Dr. Tedros Adhanom Ghebreyesus, director general de la Organización Mundial de la Salud "es uno de los riesgos sanitarios más urgentes de nuestra época y amenaza con echar por tierra un siglo de progreso médico"(5).

Hoy en día, más del 50\% de los antibióticos se usa de manera incorrecta en muchos países. Por ejemplo, para el tratamiento de virus (como es el caso de más del 90\% de las gripes comunes), la administración de antibióticos resulta inútil y solo aumenta la resistencia mediante la aparición de las denominadas "superbacterias". En efecto, tal es el alcance del problema de resistencia a los antimicrobianos, que se relaciona fuertemente con al menos seis de los diecisiete objetivos del desarrollo sostenible $(6,7)$.

La automedicación es una práctica frecuente en nuestro medio y en el mundo; se ha referido que la prevalencia de automedicación constituye un 90\% en India (8), 72,5\% en Murcia-Espańa (9) y 62,9\% Mansoura-Egipto (10). La automedicación en el ámbito latinoamericano es de 97.6\% en Costa Rica (11), 92,8\% en Itapua-Paraguay (12), 90,1\% Ate-Perú (13), 87\% en Venezuela, 83\% en Argentina, $73 \%$ en Colombia (14), en Chile $72 \%$ (15), asimismo en el contexto nacional 87.61\% en Mérida, Yucatán (16).

De acuerdo con una encuesta telefónica, realizada por el Gabinete de Comunicación Estratégica, nueve de cada diez encuestados (90.6\%) afirmaron que, en México, se recurre frecuentemente a la automedicación (17). En ocasiones, algunas personas se basan en sus propias experiencias (conocimiento empírico), ignorando los riesgos que implica el automedicarse, tales como la intoxicación medicamentosa, al consumir mayor dosis de la requerida, o el no logro del efecto esperado, al tomar una dosis inferior a la indicada. Específicamente el consumo de antibióticos sin prescripción médica genera resistencia bacteriana (18)

La automedicación responsable y con conocimiento, podría ser ventajosa para el primer nivel de atención en salud; esto si la población fuese apropiadamente conocedora de las indicaciones, contraindicaciones y riesgos de la automedicación. Un estudio en la población de San Cristóbal de las Casas, Chiapas, México demostró que las personas no están debidamente informadas y un alto porcentaje no lee las instrucciones especificadas en los medicamentos que consumen (19). Aunque a veces se ven obligados a recurrir u optar a la práctica de automedicación por causas diversas como son: bajos recursos económico, desempleo o carencias del sector salud (20).
Este estudio se interesó por identificar el nivel de conocimiento básico y las características de la automedicación en consumidores de medicamentos genéricos en farmacias de un municipio mexicano.

\section{MÉTODOS}

Se realizó un estudio exploratorio descriptivo y transversal en la población urbana residente en el Municipio de Jalpa de Méndez (87,249 habitantes) del estado mexicano de Tabasco. La muestra probabilística aleatoria simple $(\mathrm{n}=383)$ se estableció con un nivel de confianza de 95\%, margen de error de 0.5. La información se recolectó en 7 de las 10 farmacias existentes en la cabecera municipal durante los meses de julio y agosto del 2019. Como criterio de inclusión se consideró: ser usuario de los servicios farmacéuticos en Jalpa de Méndez, comprador de medicamentos genéricos, tener mayoría de edad y aceptar participar en el estudio. Los criterios de exclusión fueron: usuarios sin capacidad para comunicarse de forma verbal y escrita, y personal que labora en las farmacias participantes, aunque fuesen compradores de medicamentos.

El instrumento base fue el diseńado en Perú por Chávez Alayo et al (21), que fue modificado y adecuado para la comprensión de las preguntas para su aplicación en la población mexicana. Se realizó la prueba piloto para su validación con 90 usuarios que acudieron a las farmacias y se utilizó el Alfa de Cronbach obteniéndose un 0.84. El cuestionario mixto constó de 33 reactivos, divididos en tres bloques. El bloque uno, comprendió las preguntas de la uno a la seis, donde se incluyeron datos generales; el segundo bloque de la siete a la dieciocho relacionado sobre los conocimientos básicos acerca de medicamentos; el tercero, de la diecinueve a la treinta y tres, referente a patrones de automedicación.

Para la recolección de los datos se capacitó a tres encuestadores sobre el método de aplicación del cuestionario. En la evaluación del nivel de conocimiento se establecieron tres niveles (rango de niveles bajo, medio y alto con puntaje mínimo de 9 y un máximo de 36 dividido en 3 bloques. En el nivel bajo se valoró un rango bajo de 9-18, medio de 19-27 y alto de 28-36.

La captura y análisis de los datos se ejecutó mediante el paquete estadístico Statistical Package for Social Sciences (SPSS) versión 21, haciéndose estadísticas descriptivas en tablas de frecuencias y gráficos de barras. Para analizar las variables de estudios se utilizó correlación de Spearman. 


\section{RESULTADOS}

\section{Perfil del consumidor de medicamentos genéricos}

Se incluyeron 383 sujetos consumidores de medicamentos genéricos en el estudio, siendo en su mayoría mujeres (53\%), entre los 18 y 33 ańos de edad (53.3\%), con nivel de escolaridad alta (preparatoria $34 \%$ y profesionales $42 \%$ ), casadas (45\%), ocupadas laboralmente (52\%) y autodependientes (62\%).

La compra de medicamentos genéricos obedeció principalmente a la receta otorgada por el médico (83.3\%). En otros casos fueron los familiares quienes recomendaron qué medicamentos debían consumir (7.3\%) y en menor medidas fue el propio dispensador de la farmacia quién orientó la compra (5.7\%).

\section{Conocimiento básico de usuarios sobre las Especialidades Farmacéuticas Genéricas}

Entre los encuestados el nivel de conocimiento predominante fue el medio, con porcentaje del 67.1\% (Tabla 1). Los conocimientos básicos sobre medicamentos en la población se detallan en la Tabla 2, destacándose que el 85.6\% afirmó consumir medicamentos genéricos, el $89.1 \%$ conoce el riesgo del uso inadecuado de medicamentos y el $91.9 \%$, se abstiene de consumir bebidas alcohólicas al usar medicamento.

Tabla 1. Nivel de Conocimiento

\begin{tabular}{lll}
\hline & \multicolumn{2}{c}{ Tabla 1. Nivel de Conocimiento } \\
\hline Nivel & Frecuencia & Porcentaje \\
\hline Bajo & 69 & 18.0 \\
\hline Medio & 257 & 67.1 \\
\hline Alto & 57 & 14.9 \\
\hline Total & 383 & 100.0 \\
\hline
\end{tabular}

Fuente: Propia de los autores.

Tabla 2. Conocimientos básicos sobre medicamentos

\begin{tabular}{llllllll}
\hline \multicolumn{1}{c}{ Ítems } & Siempre & $\begin{array}{c}\text { Casi } \\
\text { siempre }\end{array}$ & A veces & $\begin{array}{c}\text { Sub } \\
\text { Total }\end{array}$ & $\begin{array}{c}\text { Casi } \\
\text { nunca }\end{array}$ & Nunca & $\begin{array}{c}\text { Sub } \\
\text { Total }\end{array}$ \\
\hline Consume medicamentos genéricos (similares) & $\begin{array}{l}49 \\
(12.8 \%)\end{array}$ & $\begin{array}{l}119 \\
(31.0 \%)\end{array}$ & $\begin{array}{l}160 \\
(41.8 \%)\end{array}$ & $\mathbf{8 5 . 6 \%}$ & $\begin{array}{l}37 \\
(9.7 \%)\end{array}$ & $\begin{array}{l}18 \\
(4.7 \%)\end{array}$ & $\mathbf{1 4 . 4 \%}$ \\
\hline Lee la fecha de caducidad del medicamento & $\begin{array}{l}139 \\
(36.3 \%)\end{array}$ & $\begin{array}{l}130 \\
(34.0 \%)\end{array}$ & $\begin{array}{l}99 \\
(25.8 \%)\end{array}$ & $\mathbf{9 6 . 1 \%}$ & $\begin{array}{l}10 \\
(2.6 \%)\end{array}$ & $\begin{array}{l}5 \\
(1.3 \%)\end{array}$ & $\mathbf{3 . 9 \%}$ \\
\hline $\begin{array}{l}\text { Lee y entiende las instrucciones del } \\
\text { medicamento }\end{array}$ & $\begin{array}{l}108 \\
(28.2 \%)\end{array}$ & $\begin{array}{l}161 \\
(42.0 \%)\end{array}$ & $\begin{array}{l}82 \\
(21.5 \%)\end{array}$ & $\mathbf{9 1 . 7 \%}$ & $\begin{array}{l}28 \\
(7.3 \%)\end{array}$ & $\begin{array}{l}4 \\
(1.0 \%)\end{array}$ & $\mathbf{8 . 3 \%}$ \\
\hline $\begin{array}{l}\text { Conoce los riesgos del uso inadecuado de los } \\
\text { medicamentos }\end{array}$ & $\begin{array}{l}100 \\
(26.1 \%)\end{array}$ & $\begin{array}{l}176 \\
(46.0 \%)\end{array}$ & $\begin{array}{l}65 \\
(17.0 \%)\end{array}$ & $\mathbf{8 9 . 1 \%}$ & $\begin{array}{l}30 \\
(7.8 \%)\end{array}$ & $\begin{array}{l}12 \\
(3.1 \%)\end{array}$ & $\mathbf{1 0 . 9 \%}$ \\
\hline $\begin{array}{l}\text { Toma precauciones cuando toma o utiliza un } \\
\text { medicamento }\end{array}$ & $\begin{array}{l}157 \\
(41 \%)\end{array}$ & $\begin{array}{l}136 \\
(35.6 \%)\end{array}$ & $\begin{array}{l}64 \\
(16.7 \%)\end{array}$ & $\mathbf{9 3 . 3 \%}$ & $\begin{array}{l}21 \\
(5.4 \%)\end{array}$ & $\begin{array}{l}5 \\
(1.3 \%)\end{array}$ & $\mathbf{6 . 7 \%}$ \\
\hline $\begin{array}{l}\text { Sabe usted que los medicamentos pueden } \\
\text { provocar reacciones adversas }\end{array}$ & $\begin{array}{l}118 \\
(30.8 \%)\end{array}$ & $\begin{array}{l}148 \\
(38.6 \%)\end{array}$ & $\begin{array}{l}86 \\
(22.5 \%)\end{array}$ & $\mathbf{9 1 . 9} \%$ & $\begin{array}{l}23 \\
(6.0 \%)\end{array}$ & $\begin{array}{l}8 \\
(2.1 \%)\end{array}$ & $\mathbf{8 . 1 \%}$ \\
\hline $\begin{array}{l}\text { Combina medicamentos con bebidas } \\
\text { alcohólicas }\end{array}$ & $2(0.5 \%)$ & $5(1.3 \%)$ & $\begin{array}{l}24 \\
(6.3 \%)\end{array}$ & $\mathbf{8 . 1 \%}$ & $\begin{array}{l}39 \\
(10.2 \%)\end{array}$ & $\begin{array}{l}313 \\
(81.7 \%)\end{array}$ & $\mathbf{9 1 . 9 \%}$ \\
\hline Sabe cómo conservar sus medicamentos & $\begin{array}{l}17 \\
(20.1 \%)\end{array}$ & $\begin{array}{l}125 \\
(32.6 \%)\end{array}$ & $\begin{array}{l}134 \\
(35.0 \%)\end{array}$ & $\mathbf{8 7 . 7 \%}$ & $\begin{array}{l}23 \\
(6.0 \%)\end{array}$ & $\begin{array}{l}24 \\
(6.3 \%)\end{array}$ & $\mathbf{1 2 . 3 \%}$ \\
\hline
\end{tabular}

Fuente: Propia de los autores. 


\section{Grupo de medicamentos genéricos que se consumen sin receta}

Los medicamentos mayormente comprados fueron los antiinflamatorios no esteroideos comúnmentedenominados AINES (46.3\%), seguido de otros (21.9\%) y de los antihistamínicos (8.1\%) (Figura 1).

El grupo de otros medicamentos comprados sin receta médica comprendió a loshemorreológicos (17.90\%), los multivitamínicos (16.70\%), antiparasitarios (15.40\%), antihipertensivo e inhibidores de la bomba de protones (12.80\%) (Figura 2).

\section{Patrón de automedicación}

La automedicación fue abiertamente reconocida por el $56.6 \%$ de los encuestados, pero el patrón de automedicación detectado se caracteriza por consumir medicamentos sin receta médica $(72.1 \%)$, por iniciativa propia (62.1\%) debido principalmente a la falta de tiempo para acudir con el médico (54.8\%), por la comodidad y fácil acceso de las farmacias $(56.7 \%$ ), y por el factor económico (bajos precios de las EFG) (49.3\%). La compra de medicamentos se orientó mayormente al tratamiento de patologías leves (70.2\%) y en su mayoría refirieron mejorar su padecimiento (74.1\%) (Tabla 3).

Se encontró una correlación de Spearman entre el conocimiento básico sobre EFG con respecto al patrón de automedicación.

\section{DISCUSIÓN}

En la población con reporte de automedicación incluida en este trabajo, sobresalieron mujeres (53\%), que además tenían conocimiento sobre las reacciones adversas que
Figura 1. Medicamentos comprados sin receta médica

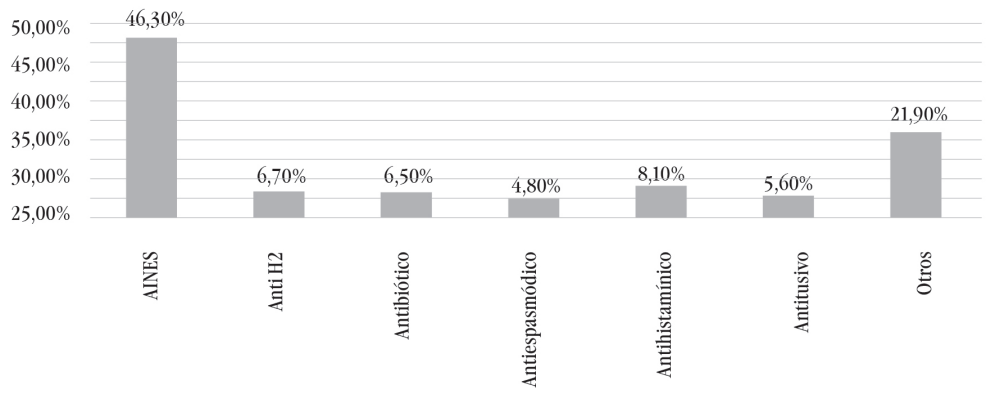

Figura 2. Otros medicamentos comprados sin receta médica

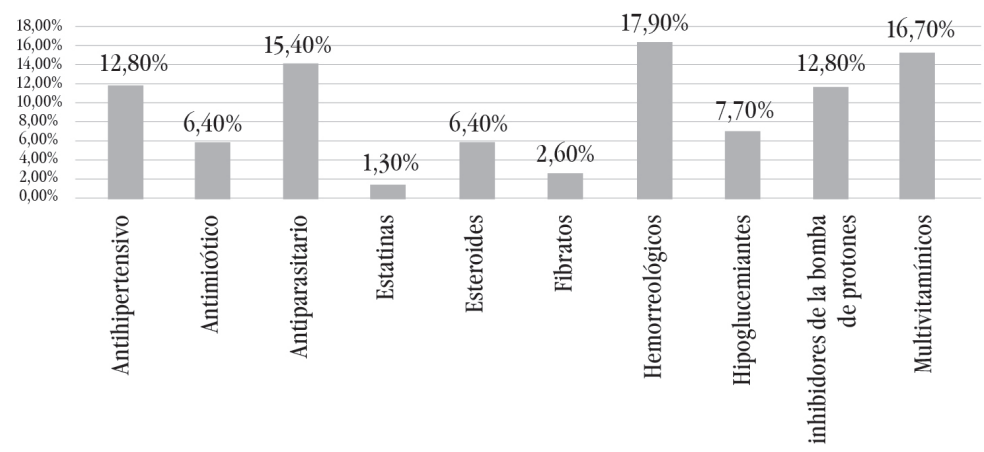

pueden ocasionar los medicamentos (91.9\%); resultados similares a los descritos en un estudio realizado en 2018 en Manabí, Ecuador; en el cual se registraron porcentajes de $57.9 \%$ (mujeres) y $82.4 \%$ (riesgos potenciales de la automedicación). 22 Este conocimiento y un alto nivel de escolaridad se correlacionó con la tendencia de la automedicación, lo cual ha sido documentado en investigaciones similares. Por ejemplo, en Trujillo Perú, el $50 \%$ de su población contaba con nivel superior y se registró un $95.8 \%$ de automedicación (23).

El porcentaje obtenido respecto a la influencia de los medios de comunicación, principalmente la televisión, sobre la automedicación (40.4\%) es similar a lo registrado en otros estudios latinoamericanos $(24,25)$. Con relación a las restricciones al consumir medicamentos, los encuestados asociaron mayormente evitar las bebidas alcohólicas en un $91.9 \%$ con la ingesta de fármacos, lo que presupone una alta responsabilidad en los consumidores, y que puede derivarse al mayor conocimiento que se tiene sobre éstos. Conductas de este tipo ya se han seńalado en una población estudiantil en Antofagasta, Chile que indicó la importancia de ingerir fármacos solo con agua (63.8\%) (26).

Los analgésicos son el tipo de medicamento utilizado con mayor frecuencia demostrados en otros estudios (27-30). Tal es el caso, que en Al Medina, Espańa (31) se reportó una cifra de $86.5 \%$ que duplica los resultados obtenido en Jalpa de Méndez (46.3\%). Estas conductas podrían explicarse por la cultura y la laxa normativa que hay en los países latinos para la comercialización de medicamentos, en muchas ocasiones incluso dispensada por personal sin formación en salud o al menos con el título de regente de farmacia.

El patrón de automedicación detectado (compra sin receta médica, por falta de tiempo para acudir con el médico, en 
Tabla 3. Patrón de automedicación

\begin{tabular}{|c|c|c|c|c|c|c|c|}
\hline Ítems & $\begin{array}{l}\text { Siem- } \\
\text { pre }\end{array}$ & $\begin{array}{l}\text { Casi } \\
\text { siempre }\end{array}$ & $\begin{array}{c}\text { A } \\
\text { veces }\end{array}$ & $\begin{array}{l}\text { Sub } \\
\text { Total }\end{array}$ & $\begin{array}{c}\text { Casi } \\
\text { nunca }\end{array}$ & Nunca & $\begin{array}{c}\text { Sub } \\
\text { Total }\end{array}$ \\
\hline $\begin{array}{l}\text { Ha consumido medicamentos sin receta } \\
\text { médica }\end{array}$ & $\begin{array}{l}17 \\
(4.4 \%)\end{array}$ & $\begin{array}{l}79 \\
(20.7 \%)\end{array}$ & $\begin{array}{l}180 \\
(47.0 \%)\end{array}$ & $72.1 \%$ & $\begin{array}{l}67 \\
(17.5 \%)\end{array}$ & $40(10.4 \%)$ & $27.9 \%$ \\
\hline $\begin{array}{l}\text { Le ha dado algún medicamento sin receta } \\
\text { médica a su hijo/a o ha sugerido a familia- } \\
\text { res, amigos/as }\end{array}$ & $\begin{array}{l}8 \\
(2.1 \%)\end{array}$ & $35(9.1 \%)$ & $\begin{array}{l}119 \\
(31.1 \%)\end{array}$ & $42.3 \%$ & $\begin{array}{l}70 \\
(18.3 \%)\end{array}$ & $\begin{array}{l}151 \\
(39.4 \%)\end{array}$ & $57.7 \%$ \\
\hline $\begin{array}{l}\text { Compra medicamentos sin receta médica } \\
\text { por iniciativa }\end{array}$ & $\begin{array}{l}10 \\
(2.6 \%)\end{array}$ & $67(17.5 \%)$ & $\begin{array}{l}161 \\
(42.0 \%)\end{array}$ & $62.1 \%$ & $\begin{array}{l}57 \\
(14.9 \%)\end{array}$ & $88(23.0 \%)$ & $37.9 \%$ \\
\hline $\begin{array}{l}\text { Compra medicamentos sin receta médica } \\
\text { por falta de tiempo }\end{array}$ & $\begin{array}{l}6 \\
(1.6 \%)\end{array}$ & $\begin{array}{l}84 \\
(21.9 \%)\end{array}$ & $\begin{array}{l}120 \\
(31.3 \%)\end{array}$ & $54.8 \%$ & $\begin{array}{l}55 \\
(14.4 \%)\end{array}$ & $\begin{array}{l}118 \\
(30.8 \%)\end{array}$ & $45.2 \%$ \\
\hline $\begin{array}{l}\text { Compra medicamentos sin receta médica } \\
\text { por motivos económicos }\end{array}$ & $\begin{array}{l}9 \\
(2.3 \%) \\
\end{array}$ & $\begin{array}{l}83 \\
(21.7 \%)\end{array}$ & $\begin{array}{l}97 \\
(25.3 \%)\end{array}$ & $49.3 \%$ & $\begin{array}{l}62 \\
(16.2 \%) \\
\end{array}$ & $\begin{array}{l}132 \\
(34.5 \%)\end{array}$ & $50.7 \%$ \\
\hline $\begin{array}{l}\text { Compra medicamentos sin receta médica } \\
\text { por comodidad y fácil acceso }\end{array}$ & $\begin{array}{l}9 \\
(2.3 \%)\end{array}$ & $\begin{array}{l}110 \\
(28.8 \%)\end{array}$ & $\begin{array}{l}98 \\
(25.6 \%)\end{array}$ & $56.7 \%$ & $\begin{array}{l}58 \\
(15.1 \%)\end{array}$ & $\begin{array}{l}108 \\
(28.2 \%)\end{array}$ & $43.3 \%$ \\
\hline $\begin{array}{l}\text { Compra medicamentos sin receta médica } \\
\text { porque considera que conoce lo suficiente } \\
\text { sobre ellas }\end{array}$ & $\begin{array}{l}14 \\
(3.7 \%)\end{array}$ & $\begin{array}{l}105 \\
(27.5 \%)\end{array}$ & $\begin{array}{l}99 \\
(25.8 \%)\end{array}$ & $57 \%$ & $\begin{array}{l}58 \\
(15.1 \%)\end{array}$ & $\begin{array}{l}107 \\
(27.9 \%)\end{array}$ & $43 \%$ \\
\hline $\begin{array}{l}\text { Compra medicamentos sin receta médica } \\
\text { porque la enfermedad es leve }\end{array}$ & $\begin{array}{l}23 \\
(6.0 \%)\end{array}$ & $\begin{array}{l}115 \\
(30.0 \%)\end{array}$ & $\begin{array}{l}131 \\
(34.2 \%)\end{array}$ & $70.2 \%$ & $\begin{array}{l}45 \\
(11.7 \%)\end{array}$ & $69(18.0 \%)$ & $29.7 \%$ \\
\hline $\begin{array}{l}\text { Compra medicamentos sin receta médica } \\
\text { porque no le gusta ir al médico }\end{array}$ & $\begin{array}{l}2 \\
(0.5 \%)\end{array}$ & $\begin{array}{l}53 \\
(13.9 \%)\end{array}$ & $\begin{array}{l}93 \\
(24.3 \%)\end{array}$ & $38.7 \%$ & $\begin{array}{l}61 \\
(15.9 \%)\end{array}$ & $\begin{array}{l}174 \\
(45.4 \%)\end{array}$ & $61.3 \%$ \\
\hline $\begin{array}{l}\text { Compra medicamentos sin receta médica } \\
\text { porque no tiene para pagar el costo de la } \\
\text { consulta médica }\end{array}$ & $\begin{array}{l}7 \\
(1.8 \%)\end{array}$ & $\begin{array}{l}46 \\
(12.0 \%)\end{array}$ & $\begin{array}{l}101 \\
(26.4 \%)\end{array}$ & $40.2 \%$ & $\begin{array}{l}61 \\
(15.9 \%)\end{array}$ & $168(43.9 \%)$ & $59.8 \%$ \\
\hline $\begin{array}{l}\text { Compra medicamentos sin receta médica } \\
\text { por influencia de la publicidad }\end{array}$ & $\begin{array}{l}3 \\
(0.7 \%)\end{array}$ & $\begin{array}{l}41 \\
(10.7 \%)\end{array}$ & $\begin{array}{l}111 \\
(29.0 \%)\end{array}$ & $40.4 \%$ & $\begin{array}{l}42 \\
(11.0 \%)\end{array}$ & $\begin{array}{l}186 \\
(48.6 \%)\end{array}$ & $59.6 \%$ \\
\hline $\begin{array}{l}\text { Cuando utilizó la automedicación, su pade- } \\
\text { cimiento mejoró }\end{array}$ & $\begin{array}{l}39 \\
(10.2 \%)\end{array}$ & $\begin{array}{l}112 \\
(29.2 \%)\end{array}$ & $\begin{array}{l}133 \\
(34.7 \%)\end{array}$ & $74.1 \%$ & $\begin{array}{l}16 \\
(4.2 \%)\end{array}$ & $83(21.7 \%)$ & $25.9 \%$ \\
\hline
\end{tabular}

Fuente: Propia de los autores.

caso de patologías leves y por los bajos costos de las EFG) es análogo al reportado en el estado de Hidalgo, México (32). El aspecto económico de la automedicación (compra de medicamentos sin receta porque no se tiene para sufragar el precio de la consulta médica) ya ha sido apuntado en otros estudios latinoamericanos (33-36).

Llama la atención que el 40\% de la población estudiada considere que los antibióticos sirven para el tratamiento de procesos virales, lo que apunta a un deficiente nivel de conocimiento y mayores riesgos para la salud, ya seńalado en otros estudios de automedicación latinoamericanos, lo que repercute en la generación de resistencia bacteriana y reduce cada día las alternativas terapéuticas cuando se presentan infecciones graves (37-40).

\section{CONCLUSIONES}

Los hallazgos en este estudio relacionan la alta escolaridad con la práctica de automedicación en la población. El patrón de automedicación detectado se caracteriza por la compra sin receta médica en el caso de patologías leves, principalmente debido a la falta de tiempo para acudir con el médico y por los bajos 
costos de los medicamentos genéricos; predominantemente analgésicos (AINES). Estos resultados fundamentan la importancia de realizar una estrategia educativa para evitar la automedicación que pone en riesgo la salud de la población.

\section{CONTRIBUCIÓN DE LOS AUTORES}

MJAC. Concepción del proyecto original, obtención de datos, interpretación de los resultados y redacción inicial del manuscrito.

HRPA. Planificación, interpretación de resultados, redacción final del manuscrito.

JACH. Interpretación estadística de los resultados.

MHMG. Interpretación de resultados.

PSJ. Obtención de datos, Interpretación de resultados.

\section{AGRADECIMIENTOS}

Ninguno.

\section{DECLARACIÓN DE CONFLICTO DE INTERESES}

Los autores declaran no tener ningún conflicto de intereses en la realización de este estudio.

\section{FINANCIACIÓN}

Ninguna.

\section{REFERENCIAS}

1. Segura-Campos LA. Medicamentos genéricos: su importancia económica en los sistemas públicos de salud y la necesidad de estudios in vitro para establecer su bioequivalencia, Costa Rica. Revista Pensamiento Actual. 2017; 17(28):108-120. DOI: 10.15517/PA.V17I28.29549

2. Torres-Serna C, Ángel-Medina JC, Klinger-Torres H, Márquez-Flórez V, Micolta JM, Sánchez-Suescún J. Medicamentos genéricos, percepción de los médicos. Cali-Colombia. Rev. Cient. Cienc. Méd. 2018; 21(1): 40-44.

3. Altamirano-Orellana V, Hauyón-González K, MansillaCerda E, Matamala-Muńoz F, Morales-Ojeda I, Maury-
Sintjago E, et al. Automedicación en estudiantes de una residencia universitaria en Chillán, Chile. Rev Cubana Salud Pública. 2019; 45(1): e1189.

4. Kregar G, Filinger EJ. żQué se entiende por automedicación? Acta Farmacéutica Bonaerense. 2005; 24(1):130-133.

5. Organización Mundial de la Salud. Ante la lentitud de los progresos realizados, la OMS ofrece una nueva herramienta y establece un objetivo para acelerar las medidas contra la resistencia a los antimicrobianos. 2019. [Internet]. (citado 16 diciembre de 2019). Disponible en: https://www.who.int/es/news-room/ detail/18-06-2019-in-the-face-of-slow-progress- whooffers-a-new-tool-and-sets-a-target-to-accelerate-actionagainst-antimicrobial- resistance.

6. Rizo-Amézquita J, Fernández SB, Lezana MI. Resistencia antimicrobiana. Boletín CONAMED. [Internet]. Ciudad de México; 18 diciembre 2018. Disponible en: http:// www.conamed.gob.mx/gobmx/boletin/pdf/boletin22/ Resistencia.pdf

7. Sebastián 0, Ashley B, Daniel E. Cuáles son las 10 principales amenazas a la salud en 2019. [Internet]. Organización Panamericana de la Salud; 2019 (citado 16 diciembre de 2019). Disponible en: https://www.paho.org/hq/index.php?option=com content\&view=article\&id=14916:t en-threats-to-globalhealth-in-2019\&Itemid=135\&lang=es

8. Venkateswarlu M, Mushtaq Pasha MA, Ebenezer I, Fatima A. A study of self medication patterns among medical students in Santhiram Medical College, Nandyal, Journal of Evolution of Medical and Dental Sciences. 2014;3(59): 13275-13281. DOI: 10.14260/jemds/2014/3767

9. Cecilia MJ, García J, Atucha NM. La automedicación en estudiantes del Grado en Farmacia. Educ Med. 2018; 19(5):277-82. DOI: 10.1016/j.edumed.2017.07.005

10. Helal RM, Abou-ElWafa HS. Self-Medication in University Students from the City of Mansoura, Egypt. J Environ Public Health. 2017; 1-7. DOI: 10.1155/2017/9145193

11. Cambronero M. Prevalencia de automedicación en estudiantes de medicina de una universidad privada de Costa Rica, 2018. [Tesis]. Universidad Hispanoamericana de Costa Rica.2018; [citado 27 de octubre de 2019]; p. 1-127. Disponible: http://13.65.82.242:8080/xmlui/ handle/cenit/3893

12. Codas M, González V, Madrazo C, Benítez G, Chamorro L, Scott C, et al. Automedicación en Itapúa: nuestra realidad. Rev. Nac. (Itauguá). 2015; 7(1):32-36. DOI: http://dx.doi.org/10.18004/rdn2015.0007.01.032-036

13. Ramos-Rueda JD. Caracterización de la práctica de automedicación en la población residente del distrito de Ate de la provincia de Lima - marzo 2014 [Tesis]. [Perú]: 
Universidad Mayor San Marcos. Facultad de Medicina; 2014. Disponible: http://cybertesis.unmsm.edu.pe/ handle/cybertesis/3647

14. López-Cabra C, Gálvez-Bermúdez J, Domínguez C, Urbina-Bonilla A, Calderón CA Vallejos-Narváez A. Automedicación en estudiantes de medicina de la Universidad del Rosario en Bogotá DC. Colombia. Rev. Colomb. Cienc. Quim. Farm. 2016; 45(3):374-384. DOI: http://dx.doi.org/10.15446/rcciquifa.v45n3.62018

15. Suqui CE. Factores asociados a la automedicación en pacientes mayores de edad que acuden al centro de salud Jima. Periodo septiembre 2017 - febrero 2018 [Tesis]. [Ecuador]: Universidad Católica de Cuenca, 2018. Disponible en: http://dspace.ucacue.edu.ec/handle/ reducacue $/ 8200$

16. Mérida-Nájera L, Durán-Gómez M, Escobar-Sánchez M, Mendoza-Godines E, Lozada-Hernández A, RomeroUreste M, Betanzos JM, Juárez-Cacahuatitla H, HernándezLópez M, Nájera-Islas B. Frecuencia de automedicación en pacientes adscritos a un hospital general de zona con medicina familiar en Hidalgo, México. Aten Fam. 2018; 25(1):12-16

17. Instituto Belisario Domínguez. Visor ciudadano No. 30 mayo 2015: Medicamentos de marca vs medicamentos genéricos, Foro económico mundial para América Latina. [Internet]. México; 2015 [citado 23 de agosto de 2019]. Disponible en: http://bibliodigitalibd.senado.gob. $\mathrm{mx} /$ bitstream/handle/123456789/2105/VC30.pdf? sequence $=1$ \&isAllowed $=\mathrm{y}$

18. Larios MJ, Sel-Escalante C, Ayudarte-Romero V. Capítulo 11: Automedicación: Fármacos más utilizados en nuestra sociedad. Molero MM, Pérez-Fuentes C, Gázquez JJ, Barragán A, África-Martos M, Simón MM. Calidad de vida, cuidadores e intervención para la mejora de la salud, Volumen 1. ASUNIVEP. Salvador: Artes Gráficas Salvador; 2017. p. 85-90. Disponible en: https:// formacionasunivep.com/Vciise/files/libros/LIBRO_3. pdf $\#$ page $=85$

19. Reyes-Guillén I, Vázquez-Gutiérrez R, Ávila-Solís F. Caracterización de la práctica de automedicación en jóvenes universitarios. Revista Digital de la Universidad Autónoma de Chiapas. 2017; 6(15). DOI: http://dx.doi. org/10.31644/IMASD.15.2017.a03

20. Tobón FA, Montoya S, Orrego MA. Automedicación familiar, un problema de salud pública. Educ Med. 2017; 158. DOI: http://dx.doi.org/10.1016/j. edumed.2017.03.004

21. Chavez-Alayo F, Mendiburu AA. Nivel de conocimientos básicos sobre medicamentos, nivel educativo y automedicación en pacientes del hospital la caleta de Chimbote. [Tesis]. [México]: Universidad Inca Garcilaso de la vega. 2016. Disponible en: https://docplayer. es/83793464-Tesis-nivel-de-conocimientos-basicossobre-medicamentos-nivel-educativo-y-automedicacionen-pacientes-del-hospital-la- caleta-de-chimbote.html

22. Ponce-Zea J, Ponce-Zea D, Rivanedeira JD. Prevalencia de automedicación: estudio exploratorio en la provincia de Manabí, Ecuador. Revista Científica Dominio de las Ciencias. 2019; 5(3): 27-41. DOI: 10.23857/dc.v5i3.922

23. Rivera-Espino M, Cabrejos JE. Automedicación en zonas urbanas y urbano marginales de la provincia de Trujillo de acuerdo a los factores socioeconómicos. Revista UCV. 2016; 8(1): 49-56.

24. Alfaro-Mora R, Monge-Guerrer A, Jerez MF, CamposCampos P, Pérez-Mora F. Características de la población universitaria que recurre a la automedicación en Costa Rica. Rev Cubana Salud Pública. 2019; 45(3): e1302.

25. Tobón FA, Montoya-Pavas S, Orrego MA. Automedicación familiar, un problema de salud pública. Revista Educación Médica. 2018; 19(2):12-127. DOI: 10.1016/j. edumed.2017.03.004

26. Valdés-González M, Salazar-Silva E, Gabino G. Comportamiento de la automedicación en estudiantes de la carrera de Química y Farmacia de la Universidad Católica del Norte. Journal of Pharmacy \& Pharmacognosy Research. 2018; 6 (5): 327-348.

27. Guerrero PA. Prevalencia de la automedicación de aines relacionada con el nivel de instrucción en sujetos de 18 a 70 ańos que acuden a las cadenas más que farmacias al sur de Quito. abril - mayo 2016. [Tesis]. [Ecuador]. Disponible en: http://dspace.uniandes.edu. ec/handle/123456789/7444

28. Sandoval-Mendoza C. Factores asociados en la automedicación de usuarios que acuden a establecimientos farmacéuticos de Santa Clara-Ate, 2017. [Tesis]. [Perú]: Universidad Cesar Vallejo, 2018 (Citado 12 de agosto de 2019). Disponible en: https://core.ac.uk/ download/pdf/225584338.pdf

29. Valencia-Alanes E, Ayaviri DE, Fernandez-Zambrana J, Camera-Rech NJ, Santos A. Características de la automedicación en el distrito 3 ciudad de Cochabamba. Revista Científica de Salud UNITEPC. 2019; 6(1):15-9. https://orcid.org/0000-0002-2250-1928

30. Cauna-Aquino V. Relación entre el nivel socioeconómico - cultural y la automedicación de pacientes con antecedentes de dolor dental que acuden al hospital Hipólitounanue de Tacna, 2015. [Tesis]. [Tacna, Perú]: Universidad Nacional Jorge Basadre Grohomann, 2015 (Citado 18 de julio 2019). Disponible en: http:// repositorio.unjbg.edu.pe/handle/UNJBG/2317

31. Mahrous, Mohamed. Frequency of use of non-prescribed medication among population sample from Al Madina 
City and its impact on quality of care in Saudi Arabia. International Journal of Health Sciences. 2018;12(5): 3-9 32. Mérida-Nájera L, Durán-Gómez M, Escobar-Sánchez M, Mendoza-Godines E, Lozada-Hernández A, RomeroUreste M, Betanzos-Pérez JM, Juárez-Cacahuatitla $\mathrm{H}$, Hernández-López M, Nájera-Islas B. Frecuencia de automedicación en pacientes adscritos a un hospital general de zona con medicina familiar en Hidalgo, México. Aten Fam. 2018; 25(1):12-16 https://doi. org/10.22201/facmed.14058871p.2018.1.62923

33. Pillaca-Medina M, Carrión-Dominquez K. Automedicación en personas adultas que acuden a boticas del distrito Jesús Nazareno, Ayacucho 2015. An. Fac. med. 2016: 77(4);387-392

34. Gómez-Gil P. Factores asociados a la automedicación con Aines en pacientes ambulatorios de la consulta externa de cirugía. [Tesis]. [Perú] Universidad Privada Antenor Orrego, 2017(consultado 17 octubre de 2019). Disponible en: http://repositorio.upao.edu.pe/ bitstream/upaorep/2723/1/RE_MED.HUMA_PAOLA. GOMEZ_AUTOMEDICACION.CON.AINES_DATOS.PDF

35. Medez DF, Vargas M, Chero-Pacheco V. Automedicación en estudiantes de enfermería en una Universidad Privada en San Juan de Lurigancho, 2017. Ágora Revista Científica. 2017;04(02):1-5. http://dx.doi.org/10.21679/arc.v4i2.87

36. Valdés-González M, Salazar-Silva E, Garrido G. Comportamiento de la automedicación en estudiantes de la carrera de Química y Farmacia de la Universidad Católica del Norte Journal of Pharmacy \& Pharmacognosy Research. 2018: 6(5);326-348

37. López C, Isabet D, Upayacu Y. Automedicación con antibióticos en trabajadores de los centros comerciales adyacentes al parque alameda de las Malvinas del cercado de Lima, 2018. [tesis]. [Perú]. Universidad privada Norbert Wiener, 2018 [consultado 17 octubre de 2019]. Disponible en: http://repositorio. uwiener.edu.pe/bitstream/handle/123456789/2692/ TESIS\%20Ricci\%20Yissela\%20-\%20Condori\%20Diana. pdf? sequence=1\&isallowed =y http:// repositorio. uwiener.edu.pe/handle/123456789/2692

38. Flores-Aranda I. Prevalencia del uso de Antibióticos en pobladores del Puerto Salaverry-Trujillo. Julio-Octubre 2018. [Tesis]. [Perú]. Universidad Católica Los Ángeles De Chimbote, 2018 [consultado 20 octubre de 2019]. Disponible en: http://repositorio.uladech.edu.pe/ bitstream/handle/123456789/7337/ANTIBIOTICOS_ PREVALENCIA_FLORES_ARANDA_INGRID_STEPHANIA. pdf?sequence $=1$ \&isallowed $=y$
39. Arevalo-Quintos A. Prevalencia del uso de antibióticos en pobladores de la Urbanización el Bosque-Trujillo. Octubre 2017 - Enero 2018. [Tesis]. [Perú]. Universidad Católica Los Ángeles De Chimbote, 2017 [consultado 21 octubre de 2019]. Disponible en: http://repositorio. uladech.edu.pe/bitstream/handle/123456789/3678/ PREVALENCIA_ANTIBIOTICOS_AREVALO_QUINTOS_ ALESSANDRA.pdf?sequence $=1$ \&isallowed $=y$

40. Bautista R. Uso y abuso de antibióticos en pacientes que asisten a consulta de medicina general, centro doctor Inocencio Diaz Pińeyro, abril-julio 2018. [Tesis]. [República Dominicana]. Universidad Nacional Pedro Henríquez Ureńa Facultad de Ciencias de la Salud, Escuela de Medicina, 2018. Disponible en: http://repositorio. unphu.edu.do/bitstream/handle/123456789/1288/ Uso $\% 20 \mathrm{y} \% 20$ abuso $\% 20 \mathrm{de} \% 20$ antibio\%cc\%81 ticos $\% 20$ en $\% 20$ pacientes $\% 20$ que $\% 20$ asisten $\% 20$ a $\% 20$ consulta $\% 20 \mathrm{de} \% 20$ medicina $\% 20$ general $\% 2 \mathrm{c} \% 20$ Centro\%20Doctor\%20Inocencio\%20Diaz\%20 Pin\%cc\%83eyro\%2c\%20abril\%20-\%20julio\%202018. pdf? sequence $=1 \&$ isAllowed $=y$ 